Brief Inductions in Episodic Past or Future Thinking: Effects on Episodic Detail and Problem-Solving

Hallford, D. J. ${ }^{1}$, Carmichael, A. $\mathrm{M}^{1}$, Austin, D. W. ${ }^{1}$, Dax, S. ${ }^{1}$, Coulston, M. I. ${ }^{1}$, \& Wong, A. ${ }^{1}$

${ }^{1}$ School of Psychology, Deakin University, 1 Gherinhap St, Geelong, Victoria 3220, Australia

\title{
Authors' contribution
}

DH designed the study with input from DA. SD, MC and AW recruited participants and collected data. AC and DH analysed the data and drafted the study protocol manuscript, and all authors contributed to redrafting. 


\begin{abstract}
Episodic specificity inductions, involving brief training in recollecting episodic details, have been shown to improve subsequent performance on tasks involving remembering the past, imagining the future and problem solving. The current study examined if specificity inductions targeting self-referential past or future episodic thinking would have dissociable effects on generating past and future episodic detail and problem solving. Sixty-three participants were randomised to either a past self-referential $o r$ future self-referential episodic induction. All participants also completed a control task. Participants randomised to the selfreferential future thinking induction generated more episodic details on past and future narrative tasks compared to a control task, whereas participants randomised to a selfreferential past thinking induction showed similar performance to the control task. When examining within-group performance of participants randomised to the past or future induction, we found some evidence of dissociable effects of inductions on narrative generation tasks, but not on problem solving outcomes. Our findings suggest that selfreferential inductions may be useful for increasing episodic specificity, but that the temporal distance and direction of the induction matters. We discuss our results in the context of the potential clinical utility of this approach for populations vulnerable to autobiographical memory disruption.
\end{abstract}

Keywords: future thinking; memory; problem-solving; episodic specificity induction. 
The ability to generate detailed and specific episodic past and future thoughts has been linked with adaptive functions such as problem-solving (Brown, Macleod, Tata, \& Goddard, 2002; Madore \& Schacter, 2014, 2016; Sheldon, McAndrews, \& Moscovitch, 2011), planning, decision-making (Dalgleish \& Werner-Seidler, 2014; Schacter, Benoit, \& Szpunar, 2017) and emotion regulation (Jing, Madore, \& Schacter, 2016; MacLeod, 2016; Taylor \& Schneider, 1989). In light of this, and the identification of psychiatric populations that show reversible shifts to less detailed episodic thinking (Berna et al., 2015; Hallford, Austin, Takano, \& Raes, 2018; Söderlund et al., 2014), data has begun to accumulate from experimental studies aimed at increasing the ability to generate self-referential detail. In the clinical field, autobiographical memory interventions have shown promise in enhancing the general ability to retrieve specific memories from the past (Takano, Moriya, \& Raes, 2017), as well as reduce symptoms of depression (Neshat-Doost et al., 2013; Raes, Williams, \& Hermans, 2009) and posttraumatic stress disorder (Moradi et al., 2014). In the experimental psychology field, episodic specificity inductions, involving brief training in recollecting specific episodic details in a laboratory environment, have been used to temporarily increase detail generation.

Studies in both healthy adult populations (Grilli, Coste, Landry, \& Mangen, 2019; Jing et al., 2016; Madore, Gaesser, \& Schacter, 2014; Madore \& Schacter, 2014, 2016) and in clinically depressed individuals (McFarland, Primosch, Maxson, \& Stewart, 2017), have demonstrated that an episodic specificity induction, involving being probed to recall details from a recently watched short movie, leads to increases in detail generation on subsequent past and future thinking tasks. Researchers have proposed that episodic specificity inductions work by biasing a person's retrieval orientation (Madore \& Schacter, 2014; Morcom \& Rugg, 2012) on subsequent task performance, whereby they focus more on episodic details relating to people, places, objects and actions. In addition to increasing detail generation, 
other outcomes that have been reported following episodic specificity inductions include improved problem-solving, more positive affect, and reduced worry (Jing et al., 2016), although subsequent studies using this movie stimulus induction have been mixed (Jing, Madore, \& Schacter, 2019). A recent study has extended seminal findings in this field by examining alternate types of episodic specificity inductions: self-referential past and future (imagination) inductions (Madore, Jing, \& Schacter, 2019). Consistent with the traditional episodic specificity induction, Madore et al. (2019) report that both types of self-referential inductions (past and future) resulted in increases in detail generation during subsequent performance on a past and future thinking task that were indistinguishable from one another. Notably, inspection of their descriptive statistics shows that the change in detail generation following self-referential inductions is considerably smaller in magnitude compared to the large effects from inductions using a standardised movie stimulus (e.g., Jing et al., 2016; Madore et al., 2014; Madore \& Schacter, 2014, 2016). This may be due to a lack of visual imagery in self-referential induction stimuli, which is associated with greater detail/vividness in mental representations (e.g., D’Argembeau \& Van der Linden, 2006), or more withinperson variation in stimuli using self-referential inductions.

Although episodic autobiographical memory and episodic future thinking have substantial overlap, distinct differences have been identified between the two forms of 'mental time travel'. While it is generally accepted that episodic memory supports future thinking by allowing people to flexibly retrieve and recombine elements of the past into novel representations of the future (Schacter et al., 2017), semantic processes (memory for facts and knowledge) are also thought to play a pivotal role in the ability to imagine the future (Irish \& Piolino, 2016). Similarly, future events range more in novelty compared to past events, whereby novelty is necessarily restricted by lived experience. Evidence for dissociable effects of past and future thinking comes from studies examining intertemporal 
decision making. For example, in delay discounting studies, whereby inductions to promote thinking about future events, relative to recent past events, reduces the rate of delay discounting (i.e., choosing immediate smaller rewards over larger later rewards) and affects subsequent health behaviours (O'Neill, Daniel, \& Epstein, 2016; Snider, LaConte, \& Bickel, 2016; Stein et al., 2016). A focus on future thinking increases one's 'temporal frame', shifting focus to longer-term outcomes, and increasing the feeling of pre-experiencing (Snider et al., 2016) and associated details (D'Argembeau \& Mathy, 2011; Snider et al., 2016). Additionally, future event simulation has been shown to improve prospective memory performance (Altgassen et al., 2015; Brewer \& Marsh, 2010). As such, it is of interest to examine whether future thinking episodic inductions differentially increase detail generation for events further into the future (longer-term outcomes) compared to closer in the future.

Beyond emerging evidence for the impact of self-referential episodic specificity inductions on past and future detail generation, little is known about the impact of such specificity inductions on other factors such as problem-solving. A particular feature of episodic future thinking is that it involves the recombination of information from memory and, therefore, often the simulation of novel conditions or circumstances (Schacter, Addis, \& Buckner, 2007, 2008). If future thinking is primed to be novel, then this might also enhance the ability to think about novel solutions or outcomes when considering future problems they could encounter. While more detailed autobiographical memory retrieval is related to problem-solving ability (Beaman, Pushkar, Etezadi, Bye, \& Conway, 2007), relatively less research has focused on detail in episodic future thinking and problem-solving. Brown et al. (2002) found that increased detail in imagining a future stressful event was related to an improved ability to simulate an effective step-by-step response. This also increased the subjective probability that the outcome would be positive. Other research has demonstrated that individuals who mentally simulate themselves engaging in a future task tend to 
experience better outcomes relative to control conditions (Taylor, Pham, Rivkin, \& Armor, 1998; Taylor \& Schneider, 1989). These studies, however, did not contrast episodic future thinking with an episodic memory condition, and investigating whether episodic specificity inductions differentially impact problem solving performance may provide insights about the mechanisms of these inductions.

Although there is an emerging body of literature examining the impact of episodic specificity inductions on subsequent task performance, studies utilising personally-relevant, self-referential inductions are more limited (Madore et al., 2019). In the current study, we compared the effects of self-referential episodic past and future thinking inductions, relative to a control task, on subsequent performance on a number of tasks, including problem solving, episodic memory and episodic future thinking. Based on previous research, we hypothesised that both past and future inductions would significantly increase detail in episodic past and future thinking. It was further hypothesised that future thinking inductions (compared to past thinking inductions) would bolster the ability to generate episodic details about a novel event further in the future (Snider et al., 2016). We also hypothesised that both inductions would increase relevant problem-solving steps proposed to solve a worrisome problem and positive expectancies relating to the outcomes of problems.

\section{Method}

\section{Participants}

Our final sample included 30 participants randomised to a future thinking induction (age range $=18-59, M_{\text {age }}=25.67, S D_{\text {age }}=9.09,44.7 \%$ female) and 33 randomised to a past thinking induction (age range $=18-54, M_{\text {age }}=25.55, S D_{\text {age }}=8.16,63.6 \%$ female). Groups did not differ in terms of age or sex (age: $t(61)=.056, p=.96$; sex: $\chi^{2}(2)=3.19, p=.20$ ). We recruited participants through social media advertisements on Facebook and snowballing, 
whereby participants were also asked to distribute an invitation among their personal networks.

The inclusion criteria required participants to be aged 18 years or over and Englishspeaking due to the language demands of tasks. The project was approved by the University Human Research Ethics Committee and informed consent was gained from all participants prior to participation. Participants received a $\$ 15$ retail gift voucher for their time and participation.

\section{Procedure Overview}

Participants followed a link to the Qualtrics online survey platform which hosted the study baseline materials. Participants were first shown a screen with a plain language statement about the study and provided their informed consent. They then completed a baseline demographic questionnaire and provided problems for the problem-solving task utilised in the study. After completing all baseline materials, participants were automatically randomised to an induction condition (past or future). The first in-person testing session occurred between 0-8 days after the baseline questionnaire was completed $\left(\mathrm{M}_{\text {days }}=3.38\right.$, $\left.\mathrm{SD}_{\text {days }}=1.89\right)$, and the second occurred 1-8 days after the first session $\left(\mathrm{M}_{\text {days }}=4.63, \mathrm{SD}_{\text {days }}=\right.$ 1.58). In each in-person testing session, participants either completed a control induction or the episodic specificity induction they were randomised to at baseline (past or future). If they completed the control induction in the first session, they then completed experimental induction in the next session (or visa-versa). The order or induction (control vs experimental) was counterbalanced across participants. In each session, participants also completed all key outcome tasks including generating self-referential past and future narratives in response to cue words and completing a problem solving task. Each session took approximately one hour and was conducted with a researcher in the participants' own home in a quiet space free from distraction. 


\section{Materials}

Episodic Specificity Inductions. For the episodic thinking induction participants were asked to think about two self-referential events that could occur in their future or had occurred in their past, dependent on which episodic thinking condition they were randomized to. They were instructed to think about events occurring in the space of a day and in a particular place, and to provide as much detail as they could. We prescribed the events, their timeframe, and their emotional valence to control for heterogeneity in the content. In the future condition, participants were instructed to think of two events that they might be personally be involved in: a positive experience on holiday in a place they had never been to before in approximately one year's time, and a negative experience during a first day at a new job in approximately three years' time. We chose these timeframes as we wanted to promote novel thinking in the future condition, as events further in the future are associated with less familiarity (D'Argembeau \& Van der Linden, 2004). For the past condition, the events were a positive experience on a birthday from approximately 3 years ago, and a negative experience of a day of injury or illness approximately one year ago. All participants were able to recall such experiences from their past. The order of presentation of these prescribed events was counterbalanced across the sample. Once participants understood the task they were probed for specific episodic details about the event for a total of five minutes using a standardized script adapted from Jing et al. (2016). Prompts included the environment the narrative occurred in, sensory experiences, the people that were present, and the sequence of events. If participants strayed from discussing the prescribed event, they were reminded to keep the event details to the particular day and place. Then they rated the novelty or familiarity of the contents that came to mind for each of the two events using a scale from 1 (seemed familiar like something that has already happened) to 9 (seemed like something new and completely novel). 
Control Induction. Similar to the protocol of Madore et al. (2019), the control condition involved an effortful task that was unrelated to episodic thinking: a packet of math problems equivalent to a sixth grade level difficulty. They were given 15 minutes, so as to match the time taken in the episodic induction to establish the events to be used (estimated at five minutes total) and describe the events (10 minutes). Participants were advised to concentrate on the maths problems for the duration, but that it was not expected they would complete all of them, nor would their scores be used in the study.

Generation of Past and Future Narratives. To assess participants' ability to generate past and future self-referential narratives, a researcher presented participants with cue words and instructed them to describe an event that had occurred in the past or could occur in the future. The event did not have to directly relate to the cue word. Four concrete noun cue words were used after the control or induction, two relating to the past and two relating to the future. The cue words were taken from (Addis, Sacchetti, Ally, Budson, \& Schacter, 2009) and included car, shoes, stain, tower, oven, engine, arm, baby. In order to examine the impact of temporal distance of episodic thought, participants were asked to provide one event from the next and past few weeks, and one from the next and past few years. A different set of cue words were used for the control and induction conditions, and the order of presentation (future narrative cues first or past narrative cues first) was counterbalanced across participants. We instructed participants to choose events that they were/would be personally involved in, that occurred within a 24 hour timeframe, and to describe it in as much detail as possible within a three-minute time limit. If participants provided unrelated commentary or details, or an event lasting more than a day they were reminded a maximum of two times to discuss the specific event.

Coding of past and future narratives. Responses were audio recorded, transcribed and coded following the Autobiographical Interview (AI) scoring protocol (Levine, Svoboda, 
Hay, Winocur, \& Moscovitch, 2002) to assess internal (episodic) and external (non-episodic) details generated. Transcribed narratives were broken down into distinct parts of information bits categorized as internal/episodic information relating to the event (e.g., event unfoldings, spatio-temporal information, perceptual information, and thoughts/emotions) or external/non-episodic information (e.g., details extraneous to the main event generated, semantic information not specific to time and place, repetition of information, metacognitive statements). To establish inter-rater reliability, four trained raters, blind to the task and condition, coded 25 responses to the $\mathrm{AI}$ (AW, MC, NR \& SD). The inter-rater reliability for the episodic and non-episodic details was found to be acceptable (ICC $=.80$ and .75 , respectively). The remaining set of responses were then scored by one of the raters. As we were primarily interested in how many episodic (internal) details a person generated in their narratives based on temporal distances (past/next few weeks, and past/next few years) we ended up with four key outcome variables: internal past details (weeks), internal past details (years), internal future details (weeks), internal future details (years). For completeness, we also calculated these same outcomes for external details.

Problem-Solving Measure. Problem-solving was assessed using the Means-End Problem Solving procedure (MEPS; Platt \& Spivack, 1975 ), modified as per Jing et al. (2016). Prior to the experiment commencing, participants were asked to provide information about 15 potential worrisome events/problems that could occur to them in the near future (3-6 months), and a goal or end-state that was desired. These problems were to be realistic, and to have the possibility of real outcomes in their life. They provided information relating to what the problem was, what is it that worried them about it, and what the good or bad outcomes were that could occur. Participants were asked for three problems in each domain of social/personal relationships, work/study, health, finances, and "other problems not yet considered' and to rate their level of anxiety or worry about each problem on a scale from 1 
(not at all) to 9 (very much so). Participants were followed up if their problem was difficult to understand, seemingly unsolvable, or if they provided problems that caused very low levels of anxiety. Two problems from each of the domains were randomly selected, and then one of each of these were randomly selected for either the control task or episodic induction. This equated to five problems to be solved after the control task or induction.

When administered after the control task or episodic thinking induction, participants had three minutes to provide as many steps as they could to solve this problem and reach the goal or end-state they had nominated. They were prompted at the start of the task to provide as much detail as possible. The problems were presented on a laptop computer and the responses were typed. The responses were scored for the number of relevant steps, as defined as a behaviour that would be effective in leading toward the stated goal. As above, an initial subset of 10 participants' responses were rated by all four of the raters, and inter-rater reliability was found to be good $(\mathrm{ICC}=.93)$. These four raters then coded the remaining responses, only coding participants they had not personally tested while blind to the task and condition. Scores for relevant steps for the five problems were averaged for each participant at each task, and the internal consistency of these items was good (average Cronbach's $\alpha$ $=.84$ across the tasks).

Problem-related subjective measures. After providing relevant steps to each of the problems, the participants rated 1) how anxious or worried they were about the problem, 2) how likely it was that they would experience a good outcome for the problem, 3) how likely it was that they would experience a bad outcome, and 4) how difficult it would be to cope with a bad outcome. The items were taken from Jing et al. (2016), who modified them from Brown et al. (2002). Responses were provided on a single-item scale from 1 (not at all) to 9 (very much so). The respective items were averaged within the task for each condition and showed an acceptable average internal reliability across the tasks (average Cronbach's $\alpha$ for 
anxiety or worry $=.77$, likelihood of good outcome $=.75$, likelihood of bad outcome $=.75$, and difficulty coping with bad outcome $=.64)$.

Consistent with Jing et al. (2016), the participants completed a coping inventory (the brief COPE; Carver, 1997) from which composite scores were calculated by averaging 14 items related to engagement coping (i.e., positive reinterpretation and growth, use of instrumental and emotional social support, active coping, planning) and 12 items related to disengagement coping (i.e., mental and behavioural disengagement, denial). The participants were asked to answer these questions in relation to how they would cope with the problems that were described in the MEPS if they actually occurred in the future, rather than how they typically cope with problems. The internal reliability of the engagement coping and disengagement coping scales was good across the tasks (Cronbach's $\alpha$ of .84 and .75 respectively).

\section{Data Analysis}

We first examined whether there were significant effects of between-subject variables that were counterbalanced across participants on key study-outcomes. This included induction-order across sessions (i.e., control followed by induction or visa-versa), inductionvalence-order within a session (i.e., positive followed by negative induction or visa-versa), or narrative-generation-order within a session (i.e., past followed by future or visa-versa). Induction-order did not impact key outcomes, whereas induction-valence-order had a significant impact on future internal details generated, with participants retrieving more future internal details for an event in the next few years when presented with a negative followed by a positive induction $(p=.002)$. Narrative-generation-order also significantly affected future episodic details generated, with participants retrieving more details for a future event in the next few weeks following an induction when they completed a past narrative task followed by the future narrative task $(p=.009)$. Narrative-generation-order also 
impacted performance on one outcome of the MEPS - with participants self-reporting their ability to cope with bad outcomes, after both an induction and in the control group, was higher when future narratives were generated before past narratives $(p s<.046)$. To mitigate the possible impact of counterbalancing, we controlled for relevant counterbalancing variables in subsequent analyses as detailed below.

To determine whether past or future inductions increased detail generation on selfreferential narrative tasks, we conducted a 2 (condition: control, induction) x 2 (induction type: past, future) x 2 (temporal distance of mental time travel: near, far) x 2 (narrative outcome task: past, future) ANCOVA controlling for induction-valance-order and narrativegeneration-order. We first report main effects, followed by two-way interactions, and then three-way and four-way interactions. To investigate between and within group differences further, we used post-hoc univariate analysis of covariance and paired samples t-tests with a Bonferroni correction for multiple comparisons.

Next, we conducted a series of 2 (condition: control, experimental) x 2 (induction type: past, future) mixed-design ANOVAs to examine the effect of condition and induction type on our problem solving outcome variables. When examining perceived likelihood of coping with a bad outcome, we controlled for narrative-generation-order in our analyses.

\section{Results}

\section{Generation of Past and Future Narratives Following Self-Referential Inductions:}

\section{Internal details}

When examining whether past or future self-referential inductions increased detail generation on narrative tasks, we first found a main effect of narrative task with participants generating more details about past events compared to future events $(F(1,59)=6.87 p=.01$, $\eta_{p}{ }^{2}=.10$; see Figure 1). We did not find a main effect of induction type, condition, or temporal distance of narrative on the number of internal details generated overall (induction 
type: $F(1,59)=.006 p=.95$; condition: $F(1,59)=1.54 p=.22$; temporal distance of narrative: $F(1,59)=.025, p=.88)$.

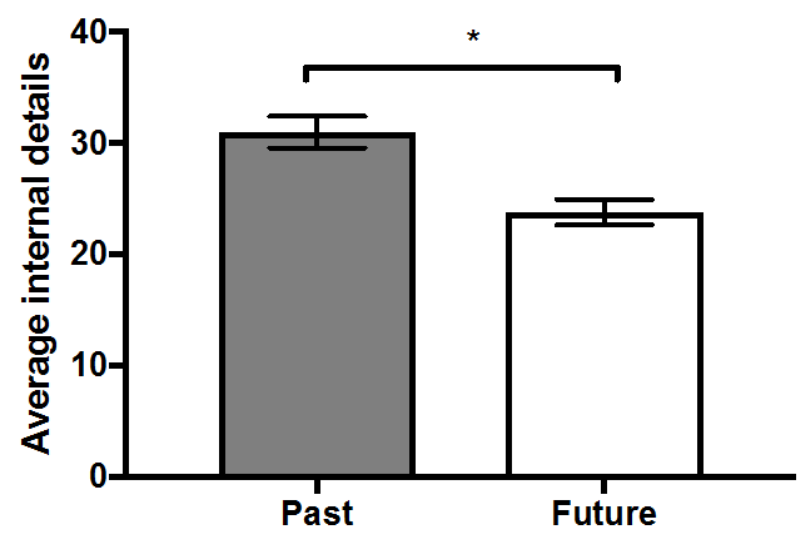

Narrative Task

Figure 1. Average internal details generated for past and future self-referential narratives collapsed across induction type, temporal distance and condition. Error bars represent standard error of the mean. $* p<.01$.

A two-way interaction between condition and induction type was detected, $F(1,59)=$ $8.16 p=.006, \eta_{p}^{2}=.12$. Follow-up tests revealed that participants in the future induction generated more internal details overall after the induction relative to the control task whereas those in the past induction generated similar in both the control and the induction condition (future induction: $t(29)=-4.45, p<.001$; past induction: $t(32)=-.63, p=.53$ ). We did not find any other significant two-way interactions (all $p \mathrm{~s}>.12$; see Figure 2). 


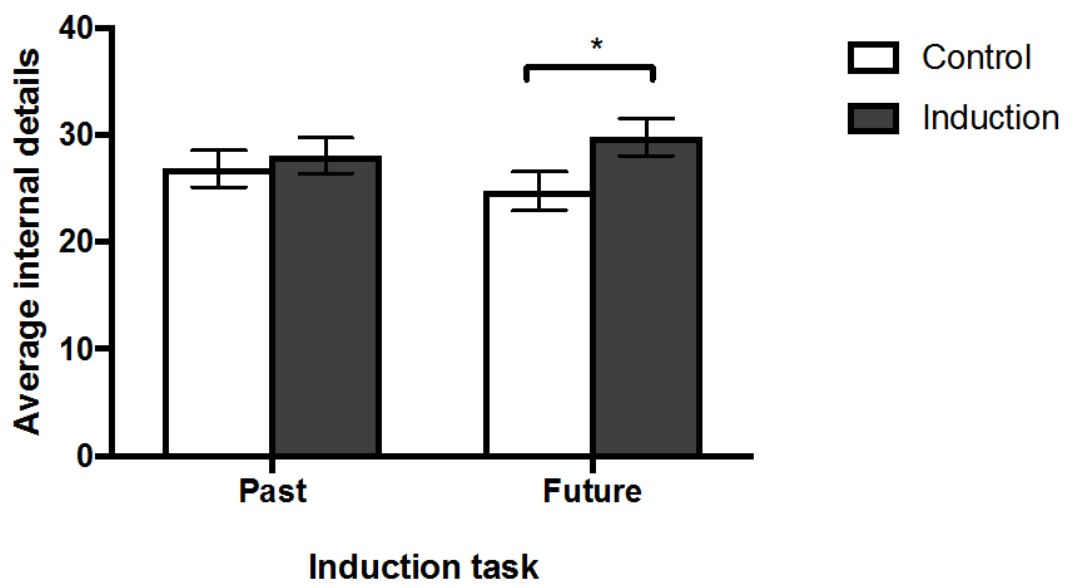

Figure 2. Average internal details generated collapsed across narrative task (past and future) following a control task (i.e., packet of math problems) versus an induction task (past or future). Error bars represent standard error of the mean. $* p<.01$.

A three-way interaction was detected between induction type $\mathrm{x}$ temporal distance $\mathrm{x}$ narrative task $\left(F(1,59)=4.11, p=.047, \eta_{p}^{2}=.07\right)$, however no other three-way interactions were significant (all $p \mathrm{~s}>.24$ ). In addition, we also found a significant four way interaction between condition $\mathrm{x}$ induction type $\mathrm{x}$ temporal distance $\mathrm{x}$ narrative task $(F(1,59)=.8 .59, p$ $\left.=.005, \eta_{p}{ }^{2}=.13\right)$. To investigate the nature of these interactions we conducted post-hoc univariate analysis of covariance to examine between group differences and paired-samples ttests to examine within group analyses.

Between-group Differences on Narrative Generation Task. When examining between-group differences, we found that when generating details for a future event further into the future (years), participants who received a future thinking induction trended towards retrieving more internal details than those in a past thinking induction, $F(1,59)=3.82, p$ $=.055, \eta_{p}{ }^{2}=.06$. No other between-group differences or trends were detected across all other key variables (all $p \mathrm{~s}>.11)$. 
Within-group Differences on Narrative Tasks. In terms of within-group differences, we examined performance of participants in the past induction and the future induction separately using paired samples t-tests. A Bonferroni correction of $p<.008$ was utilised to control for multiple comparisons.

Performance on past (autobiographical memory) narrative task. Participants in the past-induction generated more far past details following an induction task (last few years) compared to near past details following an induction $(p=.001)$ and far past details following a control task $(p=.006)$; see Figure 3. No other within-group differences survived the conservative significance threshold in the past-induction group (all $p \mathrm{~s}>.02$ ) nor were any comparisons on the past narrative generative task significant in the future-induction group (all $p \mathrm{~s}>.02)$.

Performance on future (imagination) narrative task. No within-group differences were identified on the future narrative task for participants assigned to the past-induction condition (all $p \mathrm{~s}>.49$ ), suggesting the past thinking inductions did not attenuate or bolster the ability to generate details about future self-referential narratives.

Conversely, key within-group differences were detected for participants in the futurethinking induction. Participants in the future-thinking induction generated more far future details (from the next few years) following an induction than they did for either temporal distance following a control task (near future details following a control task: $p=.001$; far future details following a control task: $p=.0002)$. Similarly, participants in the future thinking induction also generated more near future details (from the next few weeks) following an induction compared to performance following the control task (near future details following a control task: $p=.007$; far future details following a control task: $p=.005$ ). No other comparisons survived the conservative significance threshold (all $p \mathrm{~s}>.03$ ). 
Past Narrative Task

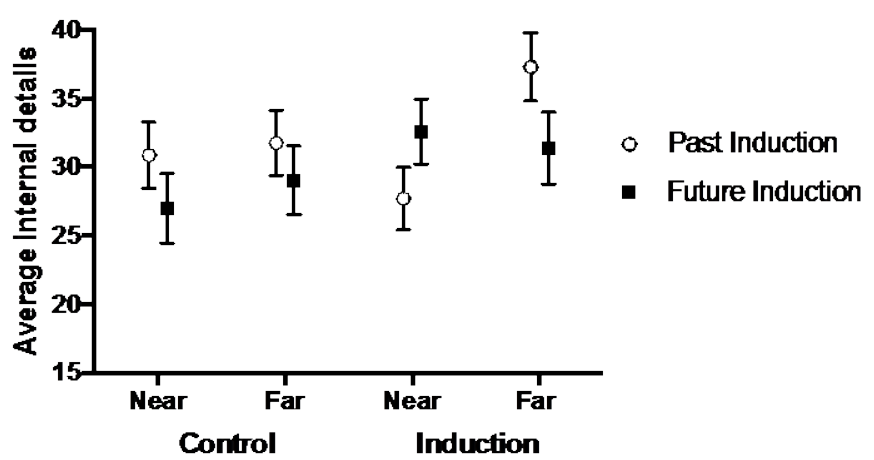

Future Narrative Task

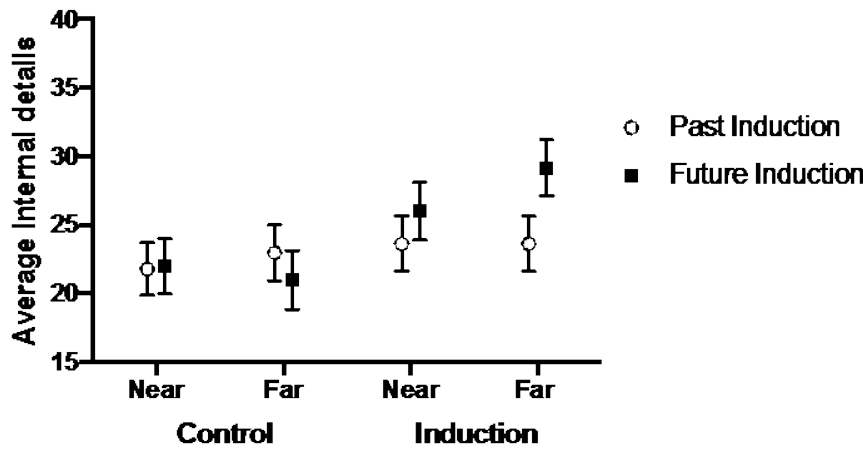

Figure 3. Total internal details generated for near and far past (left) and future (right) selfreferential narrative tasks. Error bars represent standard error of the mean.

\section{Generation of Past and Future Narratives Following Self-Referential Inductions:}

\section{External details.}

Although not central to our key hypotheses, we repeated our primary analysis to investigate whether induction type influenced generation of external details. Briefly, we did not find a main effect of induction type $(p=.59)$, nor any interactions involving induction type (all $p s>.21$ ), suggesting that induction type did not attenuate the number of external details generated across conditions or outcome task.

\section{Problem-Solving Following Self-Referential Inductions}

A mixed-design ANOVA examining the number of relevant problem solving steps did not yield a main effect of condition or induction type on the number of relevant problem solving steps provided to solve a possible problem. Furthermore, there was no condition $\mathrm{x}$ induction type interaction (condition: $F(1,61)=1.57, p=.21$; induction type: $F(1,61)=.19$, $p=.66$; condition $\mathrm{x}$ induction type: $F(1,61)=.04, p=.84)$. We used the same design to investigate the effect of condition and induction type on four single-item subjective measures related to the MEPS (namely: anxiety related to the problem/situation, perceived likelihood 
of a good outcome, perceived likelihood of a bad outcome, perceived difficulty coping with the problem/situation) finding no main effects of interactions (all $p \mathrm{~s}>.21$ ).

Next, to investigate participants rating of predicted engagement coping if selfgenerated problems were to happen, we ran a mixed-design ANOVA, revealing no main effects of induction type or condition (all $p \mathrm{~s}>.12$ ). A significant interaction between induction type $\mathrm{x}$ condition was detected $\left(F(1,61)=4.50, p=.038, \eta_{p}^{2}=.07\right)$ with post-hoc tests revealing participants in the future induction perceived they were more likely to respond to their problems using engagement coping after the induction relative to the control task. No such difference was found for participants in the past episodic thinking condition $(p=.68$; see Figure 4).

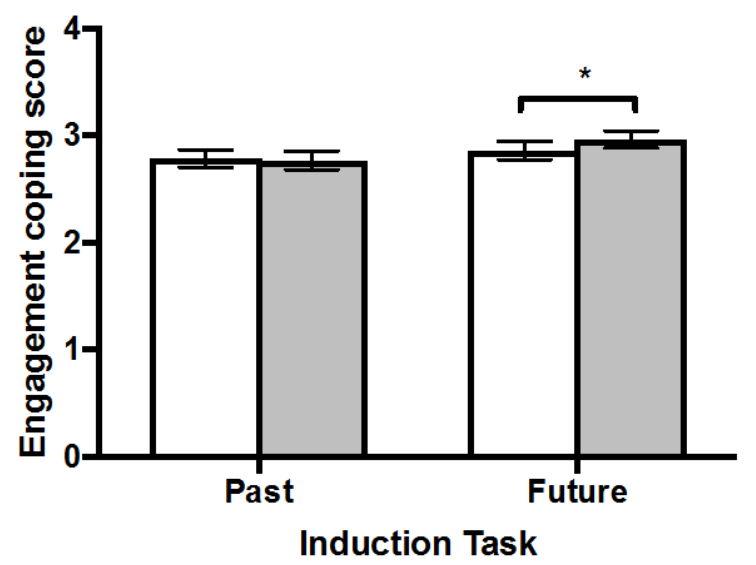

$\square$ Control Condition
$\square$ Induction Condition

Figure 4. Engagement Coping Score for participants following a control task (i.e., packet of math problems) and an episodic specificity induction (past or future). Error bars represent standard error of the mean.

The same analysis was repeated for disengagement coping, whereby a main effect of induction type was detected $\left(F(1,61)=6.90, p=.01, \eta_{p}{ }^{2}=.10\right)$. People in the future induction reported higher disengagement coping across control and induction conditions 
overall compared to those in the past induction condition (see Figure 5). We did not detect a main effect of condition or an interaction between condition $\mathrm{x}$ induction type $(p \mathrm{~s}>.90)$.

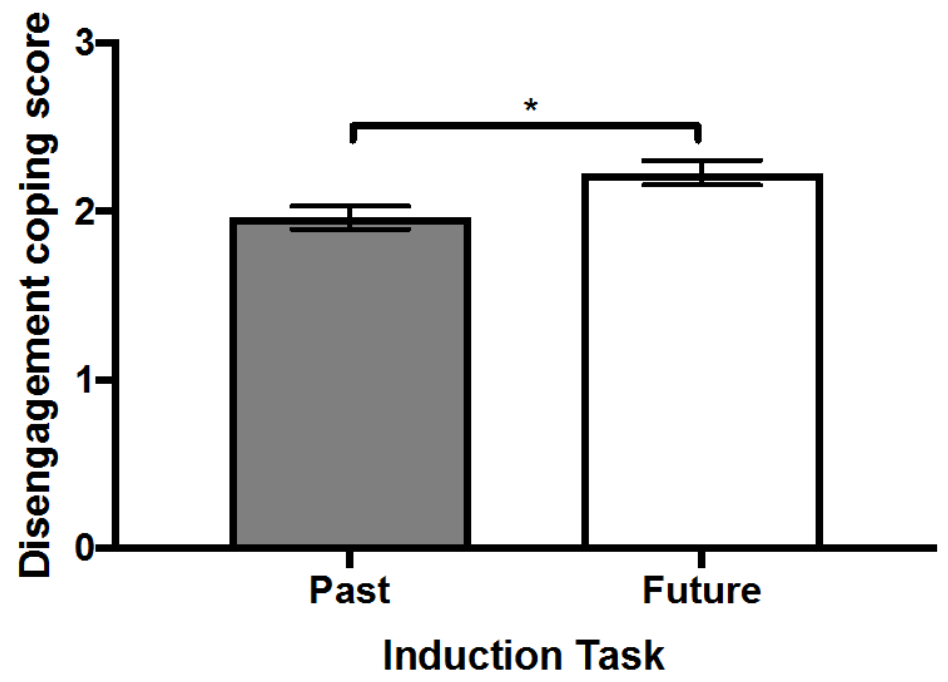

Figure 5. Disengagement Coping Score for participants following an episodic specificity induction (past or future). Error bars represent standard error of the mean.

\section{Discussion}

Although there is a growing body of literature examining the efficacy of episodic specificity inductions, few published studies have examined whether a personally-salient task can be used as an induction to bolster detail generation. The current study examined the impact of self-referential past and future episodic specificity inductions on performance on a number of tasks examining aspects of mental time travel including retrieving autobiographical memories, imaging novel events that could occur in the future, and solving hypothetical future-problems. Consistent with our hypotheses and previous research (Madore et al., 2019), we found that self-referential inductions bolstered the number of details recalled on a future-thinking task compared to a control task. In contrast to expectations, we did not find that past narratives were more detailed following an induction task. Nevertheless, examining within-induction-group performance, we found evidence that participants recalled 
more detailed narratives following an induction when the outcome task was a similar temporal distance and direction as the induction task. Finally, we did not find evidence that inductions increased problem solving ability, however, we found that our inductions appeared to modulate the subjective experience of perceived coping with future problems. Taken together, our findings do not show a universal ability for self-referential inductions to bias retrieval style towards increased detail generation, but rather our findings suggest that selfreferential inductions may be a useful method to increase detail when the induction shares similar properties to the outcome task.

Previous research using the standard episodic specific induction has involved watching and recounting details from a neutral video (Madore et al., 2014; Madore \& Schacter, 2016; Sheldon, Gurguryan, Madore, \& Schacter, 2019) whereas, similar to Madore et al. (2019), we employed self-referential past and future inductions. Unlike previous work (e.g. Madore et al., 2014; Madore et al., 2019; Madore \& Schacter, 2016), we did not find evidence that inductions increased detail generation across all tasks. We suggest that differences in how we measured past and future thinking in our task, namely our lack of visual imagery, may account for some of the differences between our findings and previous work. The only published study (to our knowledge) to date to use a self-referential episodic specific induction utilised picture cues during the outcome autobiographical memory and future thinking tasks, whereby participants had three minutes per picture to type out a detailed memory from the past or plausible future event (Madore et al., 2019). Unlike our verbal cues which were adapted from Addis et al. (2009) and tied to objects (e.g., oven, arm, baby), the cues from Madore et al. (2019) were tied to scenes and presented visually (e.g., a library or park). The importance of constructing a scene within which to situate a simulated event has been established (Hassabis \& Maguire, 2007), and it is possible that providing the scene enabled participants who had been primed to elaborate via the episodic specific 
induction to provide additional detail in Madore et al.'s study. Our findings support elements of those from Madore et al. (2019) despite our lack of pictorial cues, including evidence that inductions bolstered past or future thinking under certain temporal parameters. Specifically, a past induction resulted in more detail on a past thinking task from a few years ago, and a future thinking induction resulted in more details on a future thinking task in the next few years. Future studies should directly compare performance on cue- versus scene-based autobiographical memory and future thinking tasks following self-referential inductions to better understands the conditions under which inductions are effective.

This study is the first, to our knowledge, to investigate whether self-referential inductions could be used to increase the number of relevant steps it may take to solve a problem. Previous research using the standard episodic specificity induction have shown increased problem solving steps following the induction compared to a control condition (Jing et al., 2016; Jing, Madore, \& Schacter, 2019), which authors have described as evidence that episodic memory processes are involved in means-end problem solving. As our induction involved a generative aspect, it is possible that we may have biased our participants' orientation towards generating a future problem rather than biasing their level of detail, and future research may explore this further by directly examining the effect of different episodic specificity inductions on problem solving performance. No changes were observed between tasks on subjective reports of how anxiety-provoking the problems were, perceptions of how likely a good or bad outcome might be, or perceived difficulty coping with the problem. Previous studies employing these measures have shown mixed results, with one showing significant changes (Jing et al., 2016), and another failing to replicate this (Jing et al., 2019; notably, a follow-up study in this paper showed differences when contrasted with a control group of giving advice on the problems to another individual). However, when participants were asked how they would cope with the problems if they did occur, those who had 
undergone the future induction task reported perceiving themselves as more likely to use engagement-focused coping after the induction whereas no difference was observed in the past induction groups. Further, we found that the future induction group reported engaging in higher levels of disengagement coping in general, compared to the past induction group. Whereas the induction in previous research has involved remembering details of a video that was neutral in valence (Jing et al., 2016, 2019), our future induction required participants to project themselves into two future scenarios that were both fixed as positive and negative in valence (a positive holiday in one year; a negative experience at a job in three years). As such, it is possible that although the induction may not have increased participants ability to generate more useful steps to solve a problem, it may have primed them to consider emotional events in the future which may have modulated their perceived ability to cope with a problem in the future.

There are several methodological considerations that should be kept in mind when interpreting our findings and planning future research. The first limitation in our methodological design was our use of cue-words (adapted from Addis et al., 2009) and our restricted time frame (next/past few weeks and next/past few years) for our outcome tasks. The use of object-based cues in conjunction with restricted time frame may have caused difficulties for participants to generate a past memory that was associated with detailed recollection. We suggest that future research may prescribe a time-frame but leave the content of memories recalled unrestricted to avoid this potential limitation as per the original protocol in the Autobiographical Interview (Levine et al., 2002). In addition, directly comparing the efficacy of traditional episodic specific induction tasks and self-referential episodic specific inductions in future research could be a valuable future direction to understand any dissociable effects of induction-type. Finally, we suggest that future research may seek to code the types of details recalled on problem solving tasks to better understand whether 
inductions can bolster detail generation as well as number of effective steps taken to solve a problem similar to methodology employed by Jing et al. (2016).

Understanding the conditions under which brief episodic specificity inductions can increase episodic detail generation remains an important area of research, particularly due to potential implications in clinical populations. People with mental health conditions including depression (Raes et al., 2006; Williams et al., 2007) and post-traumatic stress disorder (Sutherland \& Bryant, 2008) have been reported to show a shift from specific to general episodic thinking, which has been associated with maladaptive outcomes (Sumner, Griffith, \& Mineka, 2010). As such, an important future direction will be investigating whether inductions can be used to increase detail of episodic future thinking and leverage adaptive outcomes associated with prospection, such as increasing goal directed behaviour and intertemporal decision making (Irish \& Piolino, 2016). We think it will be important for future research to directly compare methodologies used in both episodic specificity induction and in outcome tasks (i.e. autobiographical memory or future thinking) to better understand the generalisability and limitations of brief inductions so we can better understand their potential clinical utility. 
Funding: The authors have no funding to disclose.

Compliance with Ethical Standards: All procedures performed in studies involving human participants were in accordance with the ethical standards of the institutional research committee and with the 1964 Helsinki Declaration and its later amendments or comparable ethical standards.

Conflicts of Interest: The authors declare that they have no conflict of interest.

Informed Consent: Informed consent was obtained from all individual adult participants included in the study. 


\section{References}

Addis, D. R., Sacchetti, D. C., Ally, B. A., Budson, A. E., \& Schacter, D. L. (2009). Episodic simulation of future events is impaired in mild Alzheimers disease.

Neuropsychologia, 47(12), 2660-2671. doi:10.1016/j.neuropsychologia.2009.05.018

Altgassen, M., Rendell, P. G., Bernhard, A., Henry, J. D., Bailey, P. E., Phillips, L. H., \& Kliegel, M. (2015). Future thinking improves prospective memory performance and plan enactment in older adults. Quarterly Journal of Experimental Psychology, 68(1), 192-204. doi:10.1080/17470218.2014.956127

Beaman, A., Pushkar, D., Etezadi, S., Bye, D., \& Conway, M. (2007). Autobiographical memory specificity predicts social problem-solving ability in old and young adults. The Quarterly Journal of Experimental Psychology, 60(9), 1275-1288. doi:10.1080/17470210600943450

Berna, F., Potheegadoo, J., Aouadi, I., Ricarte, J. J., Allé, M. C., Coutelle, R., . . Danion, J.M. (2015). A meta-analysis of autobiographical memory studies in schizophrenia spectrum disorder. Schizophrenia Bulletin, 42(1), 56-66. doi:10.1093/schbul/sbv099

Brewer, G. A., \& Marsh, R. L. (2010). On the role of episodic future simulation in encoding of prospective memories. Cognitive Neuroscience, 1(2), 81-88.

doi:10.1080/17588920903373960

Brown, G. P., Macleod, A. K., Tata, P., \& Goddard, L. (2002). Worry and the simulation of future outcomes. Anxiety, Stress \& Coping, 15(1), 1-17. doi:10.1080/10615800290007254

Carver, C. S. (1997). You want to measure coping but your protocol's too long: Consider the brief cope. International journal of behavioral medicine, 4(1), 92. doi:10.1207/s15327558ijbm0401_6 
DArgembeau, A., \& Mathy, A. (2011). Tracking the construction of episodic future thoughts. Journal of Experimental Psychology: General, 140(2), 258. doi:10.1037/a0022581

D’Argembeau, A., \& Van der Linden, M. (2004). Phenomenal characteristics associated with projecting oneself back into the past and forward into the future: Influence of valence and temporal distance. Consciousness and cognition, 13(4), 844-858. doi:10.1016/j.concog.2004.07.007

Dalgleish, T., \& Werner-Seidler, A. (2014). Disruptions in autobiographical memory processing in depression and the emergence of memory therapeutics. Trends in cognitive sciences, 18(11), 596-604. doi:10.1016/j.tics.2014.06.010

Grilli, M. D., Coste, S., Landry, J. E., \& Mangen, K. (2019). Evidence that an episodic mode of thinking facilitates encoding of perceptually rich memories for naturalistic events relative to a gist-based mode of thinking. Memory, 1-7.

doi:10.1080/09658211.2019.1657461

Hallford, D. J., Austin, D. W., Takano, K., \& Raes, F. (2018). Psychopathology and episodic future thinking: A systematic review and meta-analysis of specificity and episodic detail. Behaviour research and therapy, 102, 42-51. doi:10.1016/j.brat.2018.01.003

Hassabis, D., \& Maguire, E. A. (2007). Deconstructing episodic memory with construction. Trends in cognitive sciences, 11(7), 299-306. doi:10.1016/j.tics.2007.05.001

Irish, M., \& Piolino, P. (2016). Impaired capacity for prospection in the dementiasTheoretical and clinical implications. British Journal of Clinical Psychology, 55(1), 49-68. doi:10.1111/bjc. 12090

Jing, H. G., Madore, K. P., \& Schacter, D. L. (2016). Worrying about the future: An episodic specificity induction impacts problem solving, reappraisal, and well-being. Journal of Experimental Psychology: General, 145(4), 402. doi:10.1037/xge0000142 
Jing, H. G., Madore, K. P., \& Schacter, D. L. (2019). Not to worry: Episodic retrieval impacts emotion regulation in older adults. Emotion. doi:10.1037/emo0000581

Levine, B., Svoboda, E., Hay, J. F., Winocur, G., \& Moscovitch, M. (2002). Aging and autobiographical memory: dissociating episodic from semantic retrieval. Psychology and aging, 17(4), 677-689. doi:10.1037/0882-7974.17.4.677

MacLeod, A. K. (2016). Prospection, well-being and memory. Memory Studies, 9(3), 266274. doi: $10.1177 / 1750698016645233$

Madore, K. P., Gaesser, B., \& Schacter, D. L. (2014). Constructive episodic simulation: Dissociable effects of a specificity induction on remembering, imagining, and describing in young and older adults. Journal of Experimental Psychology: Learning, Memory, and Cognition, 40(3), 609-622. doi:10.1037/a0034885

Madore, K. P., Jing, H. G., \& Schacter, D. L. (2019). Selective effects of specificity inductions on episodic details: evidence for an event construction account. Memory, 27(2), 250-260. doi:10.1080/09658211.2018.1502322

Madore, K. P., \& Schacter, D. L. (2014). An episodic specificity induction enhances meansend problem solving in young and older adults. Psychology and aging, 29(4), 913924. doi:10.1037/a0038209

Madore, K. P., \& Schacter, D. L. (2016). Remembering the past and imagining the future: Selective effects of an episodic specificity induction on detail generation. The Quarterly Journal of Experimental Psychology, 69(2), 285-298. doi:10.1080/17470218.2014.999097

McFarland, C. P., Primosch, M., Maxson, C. M., \& Stewart, B. T. (2017). Enhancing memory and imagination improves problem solving among individuals with depression. Memory \& cognition, 45(6), 932-939. doi:10.3758/s13421-017-0706-3 
Moradi, A. R., Moshirpanahi, S., Parhon, H., Mirzaei, J., Dalgleish, T., \& Jobson, L. (2014). A pilot randomized controlled trial investigating the efficacy of MEmory Specificity Training in improving symptoms of posttraumatic stress disorder. Behaviour research and therapy, 56, 68-74. doi:10.1016/j.brat.2014.03.002

Morcom, A. M., \& Rugg, M. D. (2012). Retrieval orientation and the control of recollection: An fMRI study. Journal of Cognitive Neuroscience, 24(12), 2372-2384. doi:10.1162/jocn_a_00299

Neshat-Doost, H. T., Dalgleish, T., Yule, W., Kalantari, M., Ahmadi, S. J., Dyregrov, A., \& Jobson, L. (2013). Enhancing autobiographical memory specificity through cognitive training: An intervention for depression translated from basic science. Clinical Psychological Science, 1(1), 84-92. doi:10.1177/2167702612454613

ONeill, J., Daniel, T. O., \& Epstein, L. H. (2016). Episodic future thinking reduces eating in a food court. Eating behaviors, 20, 9-13. doi:10.1016/j.eatbeh.2015.10.002

Platt, J. J., \& Spivack, G. (1972). Problem-solving thinking of psychiatric patients. Journal of Consulting and Clinical Psychology, 39(1), 148. doi:10.1037/h0033211

Raes, F., Hermans, D., Williams, J. M. G., Beyers, W., Eelen, P., \& Brunfaut, E. (2006). Reduced autobiographical memory specificity and rumination in predicting the course of depression. Journal of abnormal psychology, 115(4), 699. doi:10.1037/0021843X.115.4.699

Raes, F., Williams, J. M. G., \& Hermans, D. (2009). Reducing cognitive vulnerability to depression: A preliminary investigation of MEmory Specificity Training (MEST) in inpatients with depressive symptomatology. Journal of behavior therapy and experimental psychiatry, 40(1), 24-38. doi:10.1016/j.jbtep.2008.03.001 
Schacter, D. L., Addis, D. R., \& Buckner, R. L. (2007). Remembering the past to imagine the future: the prospective brain. Nature reviews neuroscience, 8(9), 657-661. doi:10.1038/nrn2213

Schacter, D. L., Addis, D. R., \& Buckner, R. L. (2008). Episodic simulation of future events: Concepts, data, and applications. Annals of the New York Academy of Sciences, 1124(1), 39-60. doi:10.1196/annals.1440.001

Schacter, D. L., Benoit, R. G., \& Szpunar, K. K. (2017). Episodic future thinking: Mechanisms and functions. Current opinion in behavioral sciences, 17, 41-50. doi:10.1016/j.cobeha.2017.06.002

Sheldon, S., Gurguryan, L., Madore, K. P., \& Schacter, D. L. (2019). Constructing autobiographical events within a spatial or temporal context: a comparison of two targeted episodic induction techniques. Memory, 1-13.

doi:10.1080/09658211.2019.1586952

Sheldon, S., McAndrews, M. P., \& Moscovitch, M. (2011). Episodic memory processes mediated by the medial temporal lobes contribute to open-ended problem solving. Neuropsychologia, 49(9), 2439-2447. doi:10.1016/j.neuropsychologia.2011.04.021

Snider, S. E., LaConte, S. M., \& Bickel, W. K. (2016). Episodic future thinking: Expansion of the temporal window in individuals with alcohol dependence. Alcoholism: clinical and experimental research, 40(7), 1558-1566. doi:10.1111/acer.13112

Söderlund, H., Moscovitch, M., Kumar, N., Daskalakis, Z. J., Flint, A., Herrmann, N., \& Levine, B. (2014). Autobiographical episodic memory in major depressive disorder. Journal of abnormal psychology, 123(1), 51. doi:10.1037/a0035610

Stein, J., Wilson, A., Koffarnus, M., Daniel, T., Epstein, L., \& Bickel, W. (2016). Unstuck in time: episodic future thinking reduces delay discounting and cigarette smoking. Psychopharmacology, 233. doi:10.1007/s00213-016-4410-y 
Sumner, J. A., Griffith, J. W., \& Mineka, S. (2010). Overgeneral autobiographical memory as a predictor of the course of depression: A meta-analysis. Behaviour research and therapy, 48(7), 614-625. doi:10.1016/j.brat.2010.03.013

Sutherland, K., \& Bryant, R. A. (2008). Social problem solving and autobiographical memory in posttraumatic stress disorder. Behaviour research and therapy, 46(1), 154-161. doi:10.1016/j.brat.2007.10.005

Takano, K., Moriya, J., \& Raes, F. (2017). Lost in distractors: Reduced Autobiographical Memory Specificity and dispersed activation spreading over distractors in working memory. Behaviour research and therapy, 94, 19-35. doi:10.1016/j.brat.2017.04.005

Taylor, S. E., Pham, L. B., Rivkin, I. D., \& Armor, D. A. (1998). Harnessing the imagination: Mental simulation, self-regulation, and coping. American psychologist, 53(4), 429. doi:10.1037/0003-066X.53.4.429

Taylor, S. E., \& Schneider, S. K. (1989). Coping and the simulation of events. SocialCognition, 7(2), 174-194. doi:10.1521/soco.1989.7.2.174

Williams, J. M. G., Barnhofer, T., Crane, C., Herman, D., Raes, F., Watkins, E., \& Dalgleish, T. (2007). Autobiographical memory specificity and emotional disorder. Psychological bulletin, 133(1), 122. doi:10.1037/0033-2909.133.1.122 